Case report

\title{
Intraparotid facial nerve schwannoma: A cross-country report of two cases and literature review
}

\author{
Elmira Iriskulova), Tatiana Liudchyk ${ }^{2)}$, Ulugbek Kholtoev ${ }^{3)}$, Abduavaz Ganiev ${ }^{3)}$, \\ Abdulla Abdikhakimov ${ }^{3}$, Salim Egamberdiev ${ }^{3)}$, Yulduz Khodjibekova", Sobirjon Mamarajabov ${ }^{5)}$, \\ Junichi Sakamoto ${ }^{6}$, Michitaka Honda ${ }^{7}$ \\ ${ }^{1)}$ Department of Maxillofacial Surgery, Tashkent State Dental Institute, Tashkent, Uzbekistan. \\ ${ }^{2)}$ Department of Maxillofacial Surgery, Belarusian Medical Academy of Postgraduate Education, Minsk, Belarus. \\ ${ }^{3)}$ Department of Head and Neck Tumors, Tashkent Regional Branch of the Republican Specialized Scientific-Practical \\ Medical Center of Oncology and Radiology, Tashkent, Uzbekistan. \\ 4) Department of Therapeutic Subjects No.3, Tashkent State Dental Institute, Tashkent, Uzbekistan. \\ ${ }^{5)}$ Medical pedagogical faculty, Samarkand State Medical Institute, Samarkand, Uzbekistan. \\ ${ }^{6)}$ Tokai Central Hospital, Kakamigahara, Japan. \\ ${ }^{7)}$ Department of Minimally Invasive Surgical and Medical Oncology, Fukushima Medical University, Fukushima, Japan.
}

\begin{abstract}
Intraparotid facial nerve schwannoma (FNS) is very rare disease that is difficult to diagnose; thus, there is no established treatment strategy. We herein report two cases of intraparotid FNS and highlight the diagnostic and surgical approach. The first patient presented with painful parotid mass. We performed superficial parotidectomy as a diagnostic treatment. The continuity of the nerve was interrupted when we attempted to completely resect the tumor. After resection, we carried out reconstruction of the facial nerve using neurorrhaphy with end-to-end anastomosis. A histopathological examination revealed findings of intraparotid FNS. The House-Brackmann (HB) grade was II at a 2-year follow-up examination. No recurrence has been seen in the 2 years since surgery. The second patient presented with gradually progressive swelling in the right parotid region. The patient underwent tumor removal without parotidectomy via binocular magnification and a diode laser. As a result, the patient's facial nerve function was intact at an 8-year follow-up examination (HB grade I). Tumor recurrence was not identified. Microsurgical and diode laser approaches in the management of intraparotid FNS resulted in a better overall facial nerve function without recurrence. Resection of intraparotid FNS with microsurgical and diode laser approaches should be included in a management algorithm, with prioritization of preservation of the facial nerve function.
\end{abstract}

Keywords: intraparotid, facial nerve schwannoma, laser, microsurgery, computed tomography.

(Received August 6, 2020; Accepted August 24, 2020)

\section{Introduction}

Neurogenic tumors, such as schwannomas are a very rare histological type among neoplasms of the head and neck region ${ }^{1)}$. Facial nerve schwannoma (FNS) can arise in any segment of the facial nerve from the intratemporal segment to the peripheral segment. The intratemporal segment is the most commonly involved facial nerve segment in patients with FNS ${ }^{2}$. Intraparotid FNS accounts for only 9-18\% of FNS cases ${ }^{2,3)}$.

FNSs are usually asymptomatic and are associated with slow-growing parotid swelling ${ }^{4-5}$. Although essential for treatment planning, the diagnosis of intraparotid

Corresponding author: Michitaka Honda M.D. Ph.D. Department of Minimally Invasive Surgical and Medical Oncology, Fukushima Medical University, 1 Hikarigaoka Fukushima-shi, Fukushima 960-1295, Japan. TEL: +81-24-547-1111, FAX: +81-23-547-1995, E-mail: mhonda@fukushimamed.com
FNS is difficult. Imaging studies, such as ultrasound, computed tomography (CT), and/or magnetic resonance imaging (MRI) assist in the diagnosis of patients with parotid $\mathrm{FNS}^{6-8)}$. The diagnostic value of fine needle aspiration cytology (FNAC) in the management of parotid tumors is still under debate. Previous reports have demonstrated that the histological type of parotid tumors was accurately determined by FNAC in $79.5 \%$ of cases ${ }^{9}$, and that the sensitivity of FNAC in the diagnosis of parotid tumors was $90 \%^{4)}$.

Surgical resection is useful for the diagnosis and treatment of patients with parotid tumors. However, the intraparotid FNS management decision-making process depends on various factors, including location and size, the existing preoperative facial nerve function, and patient priorities ${ }^{10-13}$. Marchioni et al. reported a high risk of facial paralysis after surgical treatment of intraparotid $\mathrm{FNS}^{10)}$. Moreover, given the benign nature of intraparotid 
FNS, preservation of the neurological function has taken precedence over tumor resection. Conservative observation without tumor resection is another treatment strate$\mathrm{gy}^{10,11,14)}$. We herein report two cases of intraparotid FNS to discuss the strategies for the diagnosis and treatment of these rare neoplasms.

\section{Case presentations}

The present study was conducted in accordance with the ethical standards of the respective committees on human experimentation (institutional and national) and with the Helsinki Declaration of 1964 and later versions. The study was approved by the Institutional Review Board of Tashkent Regional Branch of the Republican Specialized Scientific-Practical Medical Center of Oncology and Radiology and the $11^{\text {th }}$ Minsk City Clinical Hospital. We also obtained the patients' informed consent.

\section{Case 1}

A 31-year-old woman presented with a 3-year history of gradually progressive swelling in the right parotid gland region. She had a complaint of pain over the site of the swelling. Initially, there was no facial nerve dysfunction.

Physical examinations revealed a firm, immobile swelling of approximately $3.5 \mathrm{~cm}$ in the right infra-auricular region. The facial nerve function was maintained. Ultrasound demonstrated a mass lesion in the right parotid gland without regional cervical lymphadenopathy. Computed tomography (CT) showed a round, well-defined lesion measuring approximately $3.0 \times 2.0 \times 1.5 \mathrm{~cm}$ in the superficial lobe of the right parotid gland. FNAC revealed no specific malignant findings and only showed mucous material.

We performed superficial parotidectomy as a diagnostic treatment. Intraoperatively, a firm, thinly encapsulated, lobulated tumor with a smooth surface measuring approximately $3.0 \times 2.0 \mathrm{~cm}$ in size was found in the superficial lobe of the parotid gland. During the operation, we diagnosed the tumor as intraparotid FNS; however, we were unable to identify and divide the inter-layer between the main trunk of the facial nerve and the tumor. The continuity of the nerve was interrupted when we attempted to completely resect the tumor. After resection, we carried out reconstruction of the facial nerve using neurorrhaphy with end-to-end anastomosis. The histopathological examination revealed findings of intraparotid FNS. The histopathological findings, which are the spindle cells and the surrounding connective tissues were well-arranged, showed the intraparotid FNS (Fig. 1).

Postoperatively, the patient developed facial paresis (House-Brackmann [HB] grade III). The symptoms gradually improved. After 2 years of follow-up, the patient recovered to HB grade II. No recurrence was detected by a follow-up examination at 24 months after surgery.

\section{Case 2}

A 38-year-old woman presented with a 2-year history of a painless, gradually progressive swelling in the right parotid region. The patient had no pre-operative facial deficit.

A clinical examination revealed a single, firm, nontender swelling in the right parotid gland region of approximately $2.5 \times 2.0 \mathrm{~cm}$ in size. The facial nerve function was intact. CT showed a round lesion with a smooth margin measuring $2.3 \times 1.8 \times 2.6 \mathrm{~cm}$ in size within the right parotid gland deep lobe (Fig. 2). FNAC revealed pleomorphic adenoma.

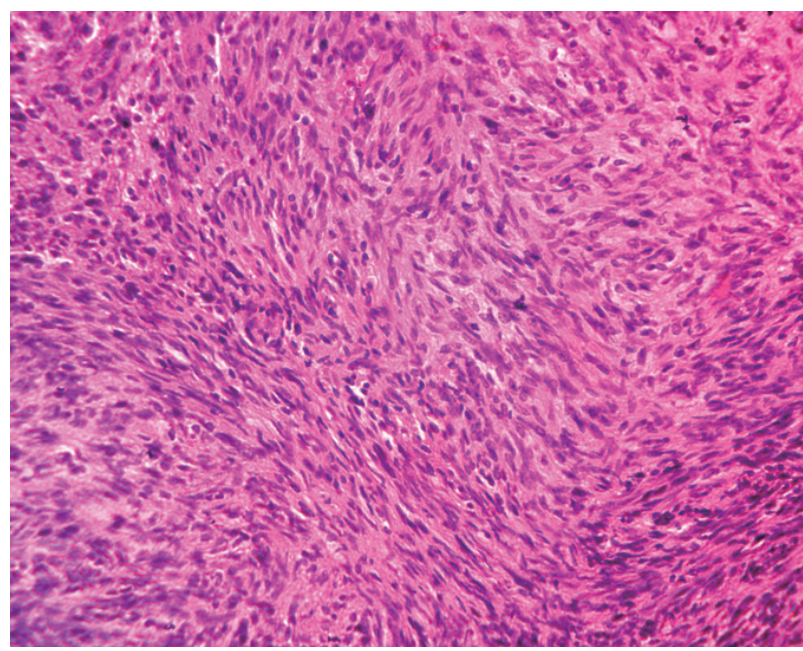

Fig. 1 Pathological findings

The microscopic findings from the specimen of case 1 showed that the spindle cells and the surrounding connective tissues were well-arranged in the Hematoxylin and Eosin Stained $(\times 400)$.

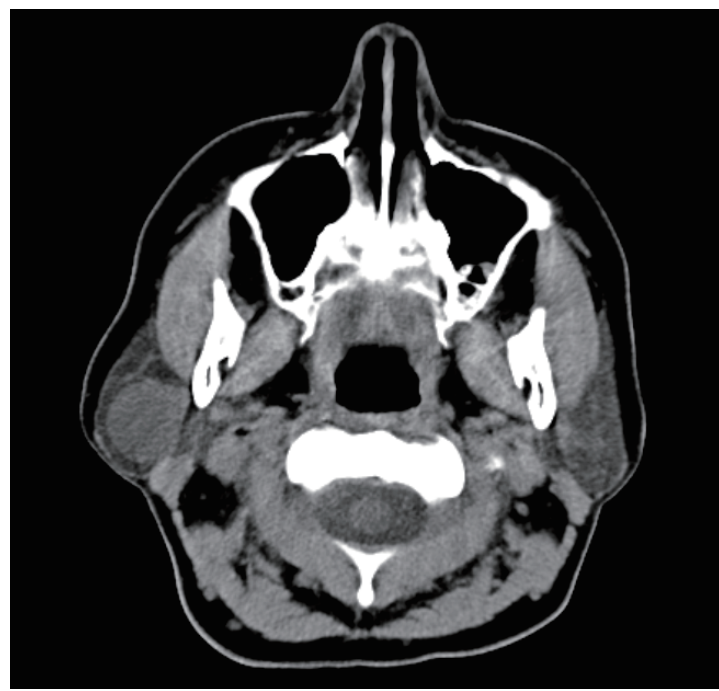

Fig. 2 The cervical CT image of case 2 Preoperative axial CT imaging showed the intraparotid facial nerve schwannoma (white arrow) in the deep lobe of the right parotid gland. 
During the operation, surgeons confirmed the wellencapsulated, nodular, $2.5 \times 2.0 \mathrm{~cm}$ tumor with approximately $1 \mathrm{~cm}$ of adhesion to the main trunk of the right facial nerve. Dissection of the facial nerve branches proceeded with the assistance of the binocular loupe with $\times 2.5$ magnification to accurately identify and preserve the facial nerve. The facial nerve branches were lifted, and the entire tumor was resected under magnification, in combination with a high-power diode laser Diolas 940-6 (Lemt, Minsk, Belarus). The laser was used in pulsed wave mode, with a flexible optic fiber of $400 \mu \mathrm{m}$, at a wavelength of $940-980 \pm 10-\mathrm{nm}$, with $3-5$ watts of power. The laser fiber was used for coagulating and minimally invasive cutting of the capsule and the intracapsular mass of the lesion. Thus, during the procedure, bipolar diathermy was not required for hemostasis. After tumor excision, the surrounding parotid tissue was treated with defocused thermolaser energy (power, 4 watts) (Fig. 3).

A probe in Stenson's duct revealed the integrity of the duct within the deep lobe of the parotid gland. Thus, tumor excision was performed without parotidectomy with preservation of the deep lobe of the parotid gland. The deep lobe of the parotid gland was also preserved in the microsurgical and laser dissection of the main trunk of the right facial nerve. At the end of surgical intervention, a superficial muscular aponeurotic system (SMAS) flap was sutured, and the skin incision was closed.

The patient had slight facial palsy after the operation. At five days after the operation, the patient's facial function had completely recovered (HB grade I). Thus, the final aesthetic and functional results were satisfactory. The post-operative clinical follow-up time was $>8$ years, there has been no evidence of recurrence or facial weakness during this time. The patient's HB grade I function has been maintained.

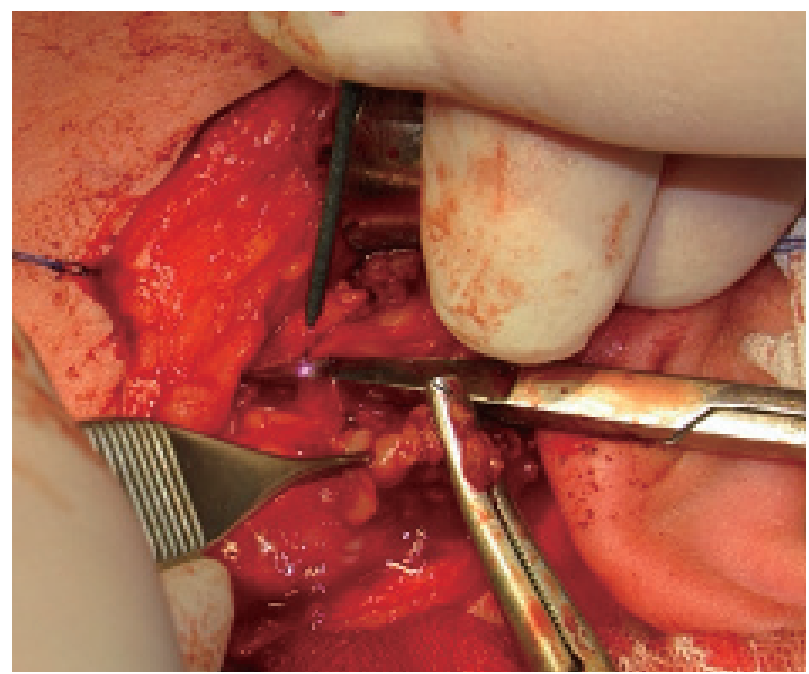

Fig. 3 Intraoperative view of the laser with flexible optic fiber

\section{Discussion}

Studies of clinical presentations of rare tumors, such as intraparotid FNSs, are necessary to make an accurate diagnosis and facilitate selection of an appropriate management strategy. Our cases were both without facial paresis, with one patient developing a painful parotid mass. The differential diagnosis of intraparotid FNS includes parotid tumors with a benign and malignant origin ${ }^{2,6)}$.

There are no specific radiological features of intraparotid FNSs. In addition, FNAC was inconclusive and with the findings confused with pleomorphic adenoma, because the findings were cytologically hypocellular and we could not definitely diagnose as the schwannoma specific spindled cells in our cases. These results can be explained by the fact that benign parotid tumors are the most common type and predominantly contain pleomorphic adenomas and Warthin's tumors ${ }^{15)}$. Thus, our two patients underwent surgical resection as a diagnostic treatment.

The various options for surgical management include superficial or total conservative parotidectomy, extracapsular excision without parotidectomy, intracapsular resection, retrograde parotidectomy, and antegrade parotidectomy $^{4,12,13,16-18)}$. The adherence of an intraparotid FNS to the facial nerve is the most frequent and essential operative findings ${ }^{5)}$. The preservation of the facial nerve function is the most relevant objective in the surgical treatment of intraparotid FNS. Microsurgery is used for precise dissection of the facial nerve during parotidectomy ${ }^{17)}$. With the microscopic approach, lasers can also be useful surgical equipment. Specifically, microsurgical and laser approaches proved to be safe and effective in surgery for schwannomas and parotid tumors ${ }^{19,20)}$.

We used a diode laser because we considered that its precision would minimize mechanical and thermic trauma to the surrounding tissues and preserve the facial nerve. As a result, the tumor was completely excised without parotidectomy. The diode laser also allowed for accurate dissection and hemostasis, limiting complications, such as post-operative salivary fistula. Overall, the patients showed a good recovery without recurrence or permanent facial nerve dysfunction.

The routine performance of parotid surgery with laser and microsurgical approaches is potentially more beneficial for facial nerve preservation; however, this is only a single report of two cases and further research is required.

\section{Conclusion}

Intraparotid FNS is a rare benign tumor that can be challenging for clinicians to diagnose and treat. Tumor resection with laser and microsurgery should be included 
in a management algorithm, with prioritization of the preservation of the facial nerve function.

\section{Acknowledgement:}

This study is supported, in part, by a non-profit organization Epidemiological \& Clinical Research Information Network.

\section{References}

1) Ma Q, Song H, Zhang P, Hou R, Cheng X, Lei D. Diagnosis and management of intraparotid facial nerve schwannoma. J CranioMaxillofacial Surg. 2010; 38(4): 271-273.

2) Carlson ML, Deep NL, Patel NS, Lundy LB, Tombers NM, Lohse CM, Link MJ, Driscol CL. Facial Nerve Schwannomas: Review of 80 Cases Over 25 Years at Mayo Clinic. Mayo Clin Proc. 2016; 91(11): 1563-1576.

3) Forton GE, Moeneclaey LL, Offeciers FE. Facial nerve neuroma: report of two cases including histological and radiological imaging studies. Eur Arch Otorhinolaryngol 1994; 251: 17-22.

4) Verma RK, Hage N, Bahl A, Bal A, Panda NK. Management Dilemmas of Intraparotid Facial Nerve Schwannoma: Report of Four Cases and Review of Relevant Literature. Indian J Surg Oncol. 2019; 10(1): 101-106.

5) Caughey RJ, May M, Schaitkin BM. Intraparotid facial nerve schwannoma: diagnosis and management. Otolaryngol Head Neck Surg. 2014; 130(5): 586-592.

6) Jaiswal A, Mridha AR, Nath D, Bhalla AS, Thakkar A. Intraparotid facial nerve schwannoma: A case report. World J Clin Cases. 2015; 3(3): 322

7) Damar M, Dinç AE, Şevik Eliçora S, Bişkin S, Erten G, Biz S. Facial Nerve Schwannoma of Parotid Gland: Difficulties in Diagnosis and Management. Case Rep Otolaryngol. 2016:1-4.

8) Shimizu K, Iwai H, Ikeda K, Sakaida N, Sawada S. Intraparotid facial nerve schwannoma: A report of five cases and an analysis of MR imaging results. Am J Neuroradiology. 2005; 26(6): 13281330.

9) Fakhry N, Antonini F, Michel J, Penicaud M, Mancini J, Lagier A, Santini L, Turner F, Chrestian MA, Zanaret M, Dessi P, Giovanni A. Fine-needle aspiration cytology in the management of parotid masses: Evaluation of 249 patients. Eur Ann Otorhinolaryngol Head Neck Dis. 2012; 129(3): 131-135.
10) Marchioni D, Alicandri-Ciufelli M, Presutti L. Intraparotid facial nerve schwannoma: literature review and classification proposal. J Laryngol Otol. 2017; 121(8): 707-712.

11) Alicandri-Ciufelli M, Marchioni D, Mattioli F, Trani M, Presutti L. Critical literature review on the management of intraparotid facial nerve schwannoma and proposed decision-making algorithm. Eur Arch Otorhinolaryngol. 2009; 266(4): 475-479.

12) Seo BF, Choi HJ, Seo KJ, Seo KJ, Jung SN. Intraparotid facial nerve schwannomas. Arch Craniofacial Surg. 2019; 20(1): 71-74.

13) Fyrmpas G, Konstantinidis I, Hatzibougias D, Vital V, Constantinidis J. Intraparotid facial nerve schwannoma: management options. Eur Arch Otorhinolayngol. 2008; 265: 699-703.

14) Shibasaki H, Tamaruya N, Nakao K. A Case of Facial Nerve Schwannoma in the Parotid Gland. Practica oto-rhino-laryngologica. Suppl., 2005; 144: 20-21.

15) Zhan KY, Khaja SF, Flack AB, Day TA. Benign Parotid Tumors. Otolaryngol Clin North Am. 2016; 49(2): 327-342.

16) Shimane T, Egawa S, Ikeda K, Kushihashi Y, Iketani Y, Takahashi G, Kawaguchi K, Ishibashi J, Kobayashi H. A case of an intraparotid facial nerve schwannoma. Stomato-pharyngology. 2017; 30(1): 91-95 (Japanese).

17) Nicoli F, D’Ambrosia C, Lazzeri D, Orfaniotis G, Ciudad P, Maruccia M, Shiun LT, Sacak B, Chen SH, Chen HC. Microsurgical dissection of facial nerve in parotidectomy: A discussion of techniques and long-term results. Gland Surg. 2017; 6(4): 308-314.

18) Anjum K, Revington PJ, Irvine GH. Superficial parotidectomy: antegrade compared with modified retrograde dissections of the facial nerve. Br J Oral Maxillofac Surg. 2008; 46(6): 433-434.

19) Mastronardi L, Cacciotti G, Roperto R, Scavo CG, Stati G. Microsurgical removal of vestibular schwannomas with flexible hand-held $2 \mu$-Thulium-fiber laser. Personal experience in 78 consecutive cases. Interdiscip Neurosurg Adv Tech Case Manag. 2019; 16: 82-86.

20) Astor FC, Hanft KL. Parotid surgery using Nd: YAG laser contact tips: clinical assessment of perioperative facial nerve function. Journal of clinical laser medicine \& surgery. 2003; 21(5): 297-299. 